A SPECTROMETER FOR MEASURING INELASTIC SECONDARIES FROM $200 \mathrm{GeV} / \mathrm{c}, \mathrm{p}-\mathrm{p}$ COLLISIONS

T. O. White

August 17, 1968

One experiment which will no doubt be a part of the early experimental program of the $200-\mathrm{GeV}$ machine will be aimed at a survey of inelastic production of secondary particles by protons to $200 \mathrm{GeV} / \mathrm{c}$ on hydrogen and heavy targets. In making a first approximation to the form which this experiment might take, it is instructive to explore in some detail the properties of a relatively cheap and simple spectrometer which can measure yields of long-lived secondaries over a wide range of longitudinal and transverse momenta.

\title{
General Layout
}

Figure 1 shows the layout of one of the simplest designs. The single-arm spectrometer is about 127 meters long. It employs two conventional 15-kG bending magnets, each 10 meters long, and a DISC counter system described below. The magnet system operates as in previous designs. ${ }^{1}$ Particles emerging from the target at a desired angle and momentum are deflected to the vicinity of point $P$ by M1. Then M2 bends the trajectory along the fixed spectrometer axis. This system accepts secondary momenta up to $180 \mathrm{GeV} / \mathrm{c}$ over the production angle range 0 to $20 \mathrm{mrad}$. 
In the absence of a restoring septum, the primary beam is, of course, deflected to various positions along an extended beam stop to the rear of the apparatus. The closest approach of the primary beam to the upstream end of the DISC counter is about $30 \mathrm{~cm}$ and occurs when $180 \mathrm{GeV} / \mathrm{c}$ positive secondaries are being analyzed at $0 \mathrm{mrad}$.

The DISC System

The DISC system envisioned consists of a scintillation counter defining telescope $\left(S_{1} \ldots S_{4}\right.$ in Fig. 1), a quadrupole triplet to render the secondary beam parallel, and two model " $\mathrm{C}$ " DISC counters ${ }^{2}$ whose specifications are given in Table I. The aperture of the system is about $10 \mathrm{~cm}$. Each quadrupole can easily be less than one meter in length, and the 10-meter length specified for each DISC counter can be reduced considerably as more efficient photocathodes are developed. The use of two DISC counters is advantageous since together they can measure each other's efficiency and provide superb background rejection. The velocity resolution of $\Delta \beta / \beta=2 \times 10^{-6}$ for one counter is sufficient for $\pi-K$ separation at $180 \mathrm{GeV} / \mathrm{c}$ where $\beta \pi-\beta \mathrm{K}=3.5 \times 10^{-6}$ and the effect of the spectrometer' $s$ finite momentum acceptance on this resolution is negligible (see below).

$\underline{\text { Resolution }}$

The intrinsic effective momentum resolution of the spectrometer is determined principally by two components: (1) the resolution due to 
secondary beam geometry and dispersion, and (2) the DISC counter velocity resolution $\Delta \beta / \beta=2 \times 10^{-6}$. Table II (a) gives $\Delta p_{1} / p$ due to (1) as a function of production angle. It is typically a few percent and can of course be reduced by replacing the defining telescope by hadoscopes, increasing the effective dispersion, etc. Qualitatively, the effective resolution tends to be dominated by (1) under the following conditions: (a) at high secondary momenta where $\Delta \beta$ brackets a wide momentum band; (b) light particles where $\Delta \beta$ corresponds to a large $\Delta p$ even at low momenta; and (c) where production angles are smaller, corresponding to a smaller beam-determined $\Delta p / p$. Some of this behavior is illustrated in Fig. 2 where the momentum bite $\Delta p$ is shown as a function of secondary momentum for $\pi^{\prime} s, K^{\prime} s$, and protons produced at $0 \mathrm{mrad}$. The dashed line indicated the effective resolution in the case of protons under the assumption that the components (1) and (2) are rms Gaussian spreads. Table II(b) contains $\Delta \mathrm{p}_{2}$ due only to the DISC counter velocity resolution for various particles and momenta at angles of 0 and $20 \mathrm{mrad}$, and Table II (c) gives the final effective resolution.

The angular resolution of the spectrometer is above $1 \mathrm{mrad}$ and the solid angle subtended is $8.2 \times 10^{-7} \mathrm{sr}$.

Comments on the Primary Beam and Target

Although a detailed description of the primary beam is beyond the scope of this note, a few comments must be made prior to rate estimates. 
Let us make the following assumptions:

(1) The momentum spread of the primary beam is $\lesssim 1 \%$.

(2) The angular divergence is $\sim 0.1 \mathrm{mrad}$.

(3) The apparent target diameter satisfies a relation (Ref. 2) which avoids erosion of the DISC acceptance:

$$
\phi<\mathrm{F} \delta \theta,
$$

where $\phi$ is the target diameter, $F$ is the focal length of the entrance quadrupole $\left(\sim 10^{4} \mathrm{~cm}\right)$, and $\delta \theta$ is the angular aperture of the DISC counter ( $10^{-4} \mathrm{rad}$ minimum).

Assumptions (1) and (2) allow us to use $\Delta p$ directly from Table II (c) in calculating rates. Assumption (3) has several implications worth noting: It allows an apparent target width of $<1 \mathrm{~cm}$. Given that the maximum angle from which the target is viewed is $20 \mathrm{mrad}$, the length is then restricted to $<40 \mathrm{~cm}$ for an actual target diameter of a few $\mathrm{mm}$. And for a tiny, target-defining beam traversing $\sim 40 \mathrm{~cm}$ of hydrogen, somewhat less than $10 \%$ of the incident protons will interact.

\section{Rate Estimates}

T. G. Walker ${ }^{3}$ has presented plots of secondary yields from 200-GeV p-p collisions as predicted in the model of Hagedorn and Ranft. 4 Based upon these yields, counting rates in this spectrometer are given in Table III. 
As a way of quickly seeing over what ranges counting rates are "reasonable", assume that $10^{8}$ protons interact in the target per machine pulse (i.e. a primary beam of $\sim 10^{9}$ protons/pulse), and that $10^{3}$ machine pulses occur per hour. Then the rates per hour of various secondaries appear in Figs. 3-5. These results indicate that even a simple spectrometer like this one can test is a very definitive way present theories of particle production.

\section{REFERENCES}

${ }^{1}$ See, for example, Ratner et al., Phys. Rev. 166, 1353, (1968).

${ }^{2}$ O. Danielsson et al., CERN/EFCA 67/16, Vol. I, p. 391.

${ }^{3}$ T. G. Walker, NAL Summer Study Report B. 5-68-24, 1968.

${ }^{4}$ R. Hagedorn and B. Ranft, CERN/EFCA 67/16, Vol. I, p. 170.

Table I. DISC Counter Specifications.

$\begin{array}{ll}\text { Length: } & 10 \text { meters } \\ \text { Aperture Diameter: } & 10 \mathrm{~cm} \\ \text { Cerenkov Angle: } & 20 \mathrm{mrad} \\ \text { Angular Acceptance: } & 1 \mathrm{mrad} \\ \Delta \beta / \beta: & 2 \times 10^{-6}\end{array}$

Table II (a). $\Delta \mathrm{p} /$ - Due to Geometry and Secondary Beam Dispersion Alone.

$\begin{array}{ccccccc}\text { Production angle, mrad } & \frac{0}{0 \mathrm{p} / \mathrm{p}(\mathrm{FW})} & \frac{5}{0.0208} & 0.0239 & 0.2080 & 0.0352 & 0.0426\end{array}$


Table II (b). $\Delta \mathrm{p}_{2}$ in GeV/c Due Only to DISC Counter Velocity Resolution.

\begin{tabular}{|c|c|c|c|c|}
\hline Momentum, GeV/c & 60 & 100 & 140 & 180 \\
\hline$\pi$ & 22.1 & 102 & 283 & 609 \\
\hline $\mathrm{K}$ & 1.77 & 8.20 & 22.5 & 47.8 \\
\hline $\mathrm{p}$ & 0.491 & 2.27 & 6.24 & 13.3 \\
\hline
\end{tabular}

Table II (c). Final Effective $\Delta p$ in $\mathrm{GeV} / \mathrm{c}$ of Spectrometer Assuming $\Delta \mathrm{p}_{1}, \Delta \mathrm{p}_{2}$ Are Gaussian Spreads.

\begin{tabular}{|c|c|c|c|c|}
\hline Momentum GeV/c & 60 & 100 & 140 & 180 \\
\hline$\pi \quad 0 \mathrm{mrad}$ & 1.25 & 2.08 & 2.91 & 3.74 \\
\hline$\pi \quad 20 \mathrm{mrad}$ & 2.54 & 4.26 & 5.96 & 7.67 \\
\hline $0 \mathrm{mrad}$ & 1.02 & 2.02 & 2.89 & 3.74 \\
\hline $\mathrm{K} \quad 20 \mathrm{mrad}$ & 1.46 & 3.78 & 5.76 & 7.57 \\
\hline $0 \mathrm{mrad}$ & 0.457 & 1.54 & 2.64 & 3.60 \\
\hline $20 \mathrm{mrad}$ & 0.482 & 2.01 & 4.31 & 6.64 \\
\hline
\end{tabular}


Table III. Yields Per Interacting Proton. (All numbers to be multipled by $10^{-7}$.)

\begin{tabular}{|c|c|c|c|c|c|}
\hline \multicolumn{2}{|c|}{ Momentum GeV/c } & \multirow{2}{*}{$\frac{60}{203}$} & \multirow{2}{*}{$\frac{100}{220}$} & \multirow{2}{*}{$\frac{140}{72}$} & \multirow{2}{*}{$\frac{180}{0.62}$} \\
\hline$\pi^{+}$ & $0 \mathrm{mrad}$ & & & & \\
\hline & $20 \mathrm{mrad}$ & 3.2 & 0.035 & & \\
\hline \multirow[t]{2}{*}{$\pi^{-}$} & $0 \mathrm{mrad}$ & 72 & 37.5 & 9.5 & 0.266 \\
\hline & $20 \mathrm{mrad}$ & 1.45 & 0.167 & & \\
\hline \multirow[t]{2}{*}{$\mathrm{K}^{+}$} & $0 \mathrm{mrad}$ & 25 & 23 & 5.9 & 0.012 \\
\hline & $20 \mathrm{mrad}$ & 0.475 & 0.0031 & & \\
\hline \multirow[t]{2}{*}{$K^{-}$} & $0 \mathrm{mrad}$ & 3.75 & 1.07 & 0.165 & 0.0018 \\
\hline & $20 \mathrm{mrad}$ & 0.237 & 0.0031 & & \\
\hline \multirow[t]{2}{*}{$\mathrm{p}$} & $0 \mathrm{mrad}$ & 43.4 & 655 & 2800 & 6240 \\
\hline & $20 \mathrm{mrad}$ & 0.6300 & 0.0558 & & \\
\hline \multirow[t]{2}{*}{$\overline{\mathrm{p}}$} & $0 \mathrm{mrad}$ & 0.289 & 0.151 & 0.0162 & \\
\hline & $20 \mathrm{mrad}$ & 0.0197 & 0.00049 & & \\
\hline
\end{tabular}




\section{FIGURE CAPTIONS}

Fig. 1. Single-arm spectrometer for inelastic p-p scattering.

Fig. 2. Contributions to momentum resolution.

Fig. 3. Pion counting rates at 0 and $20 \mathrm{mrad}$ as a function of momentum.

Fig. 4. Kaon counting rates at 0 and $20 \mathrm{mrad}$ as a function of momentum.

Fig. 5. Proton and antiproton counting rates at 0 and $20 \mathrm{mrad}$ as a function of momentum. 


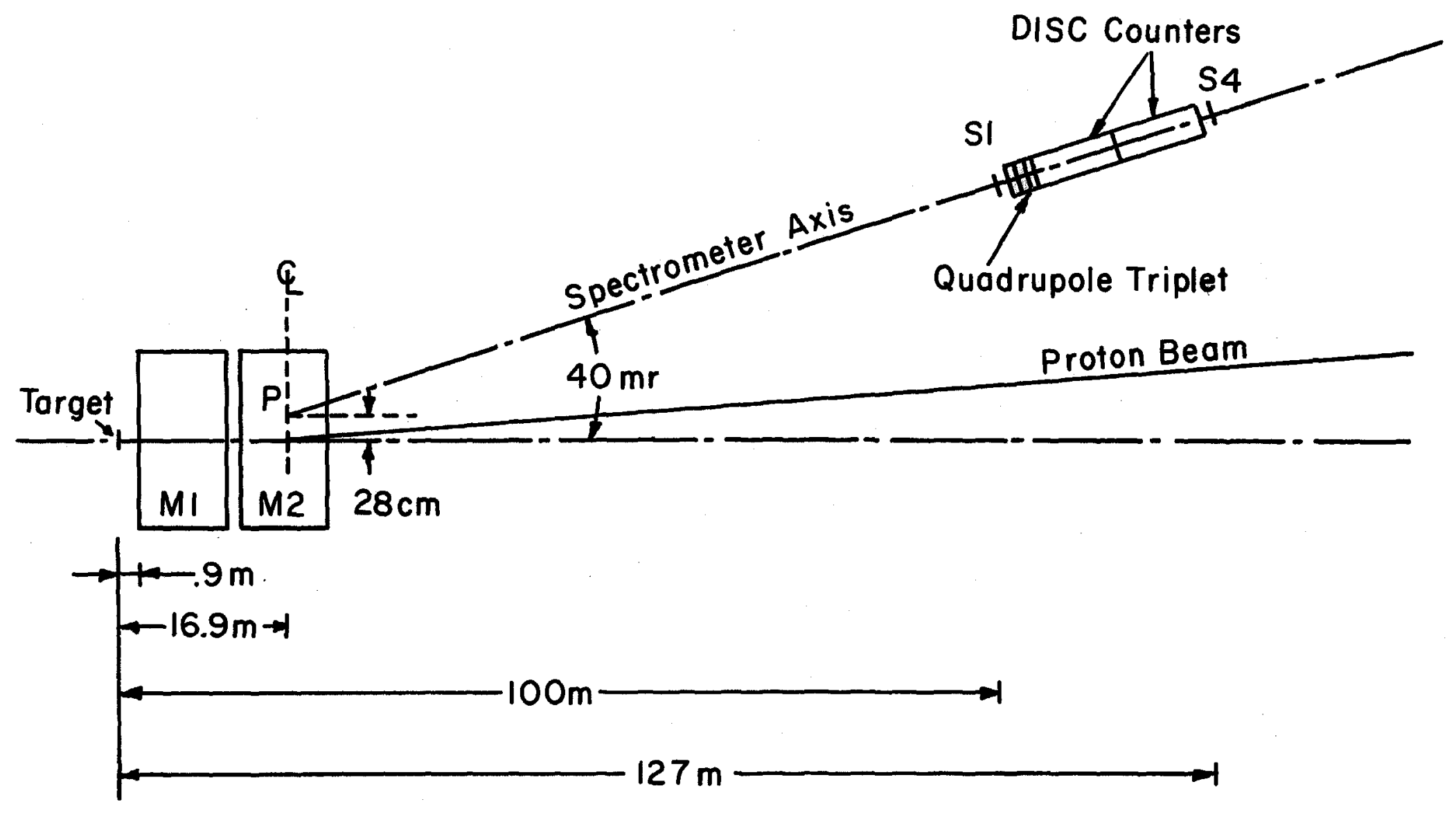

(Drawing not to Scale)

Fig. 1 


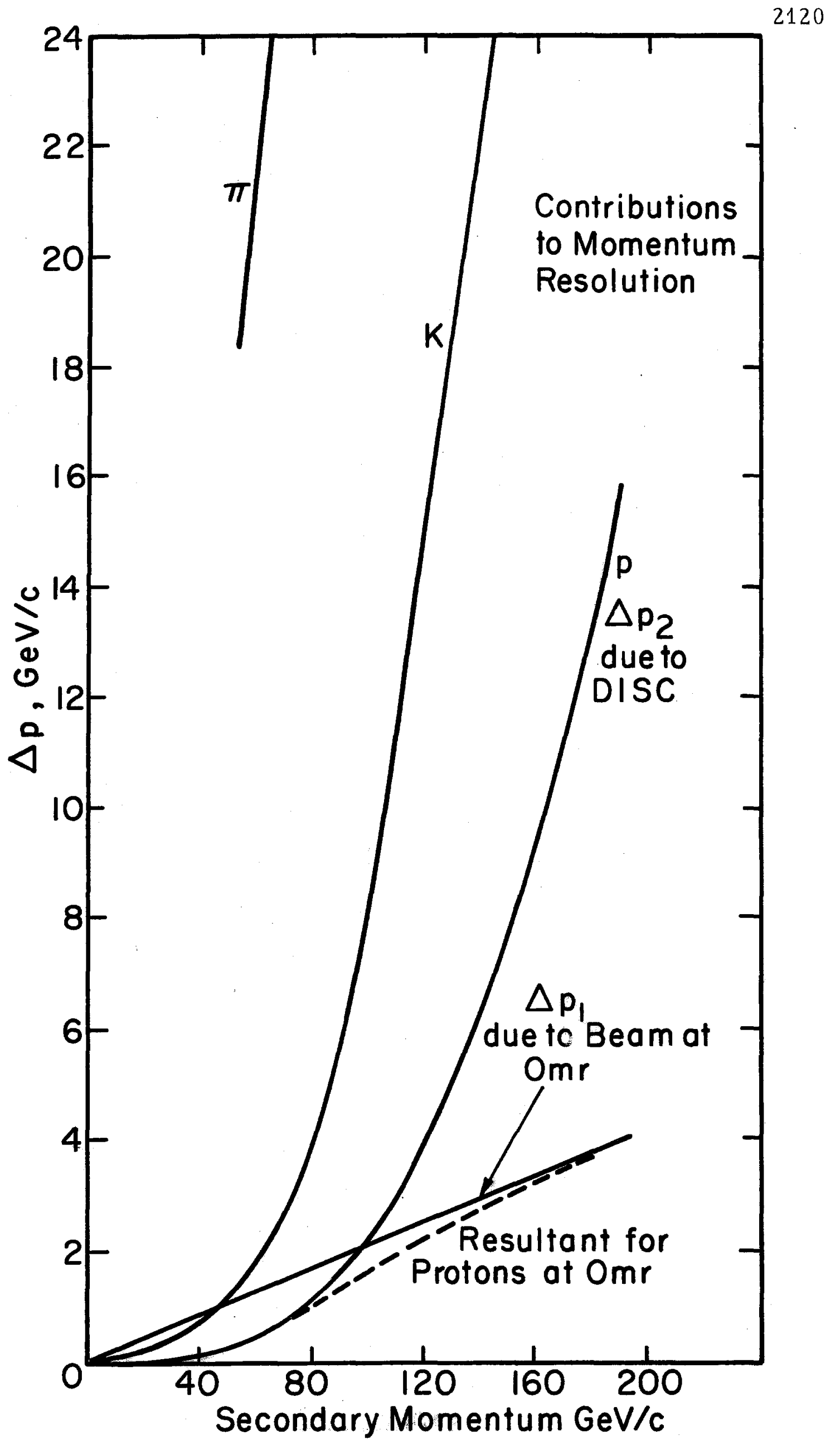




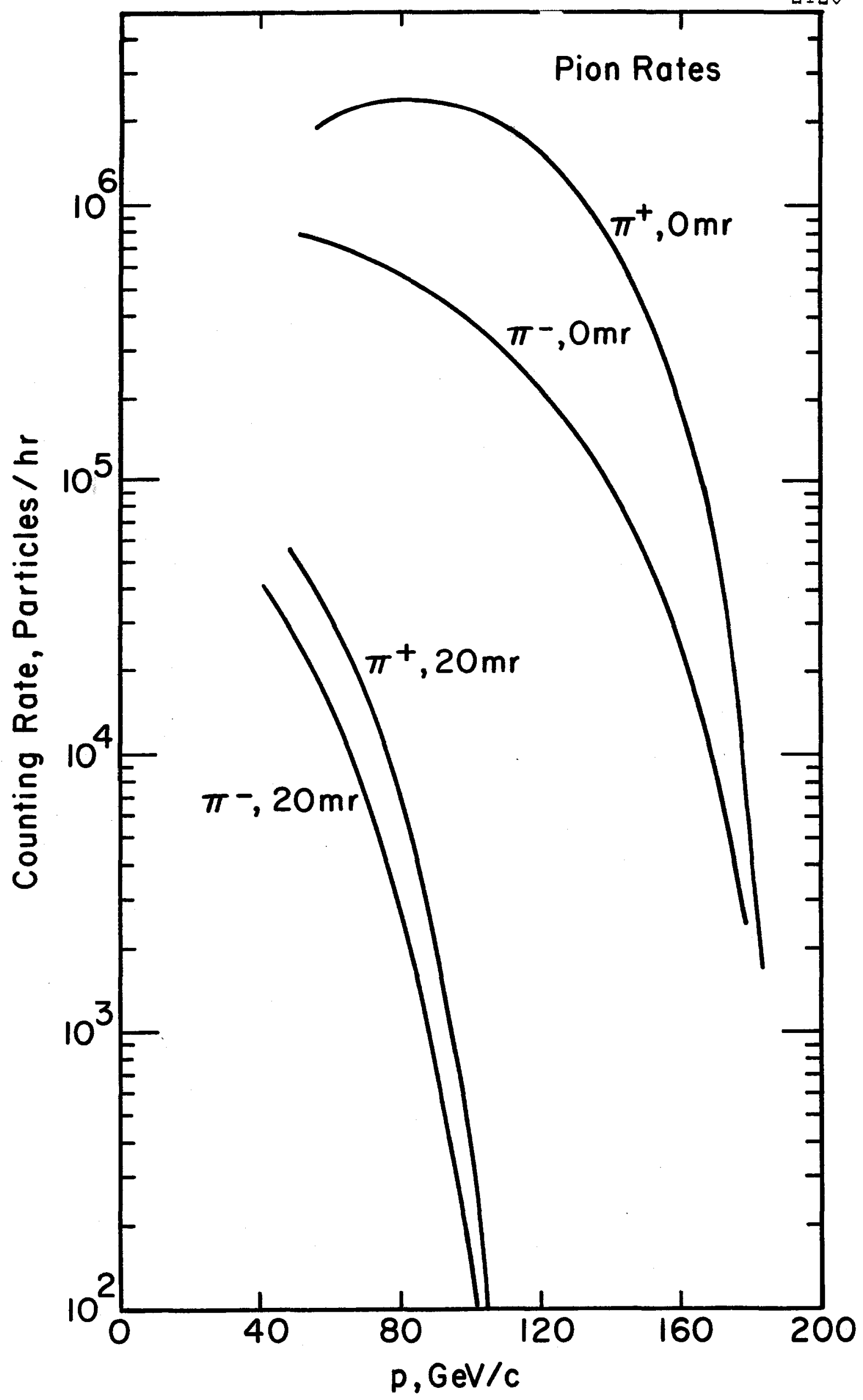

Fig. 3 


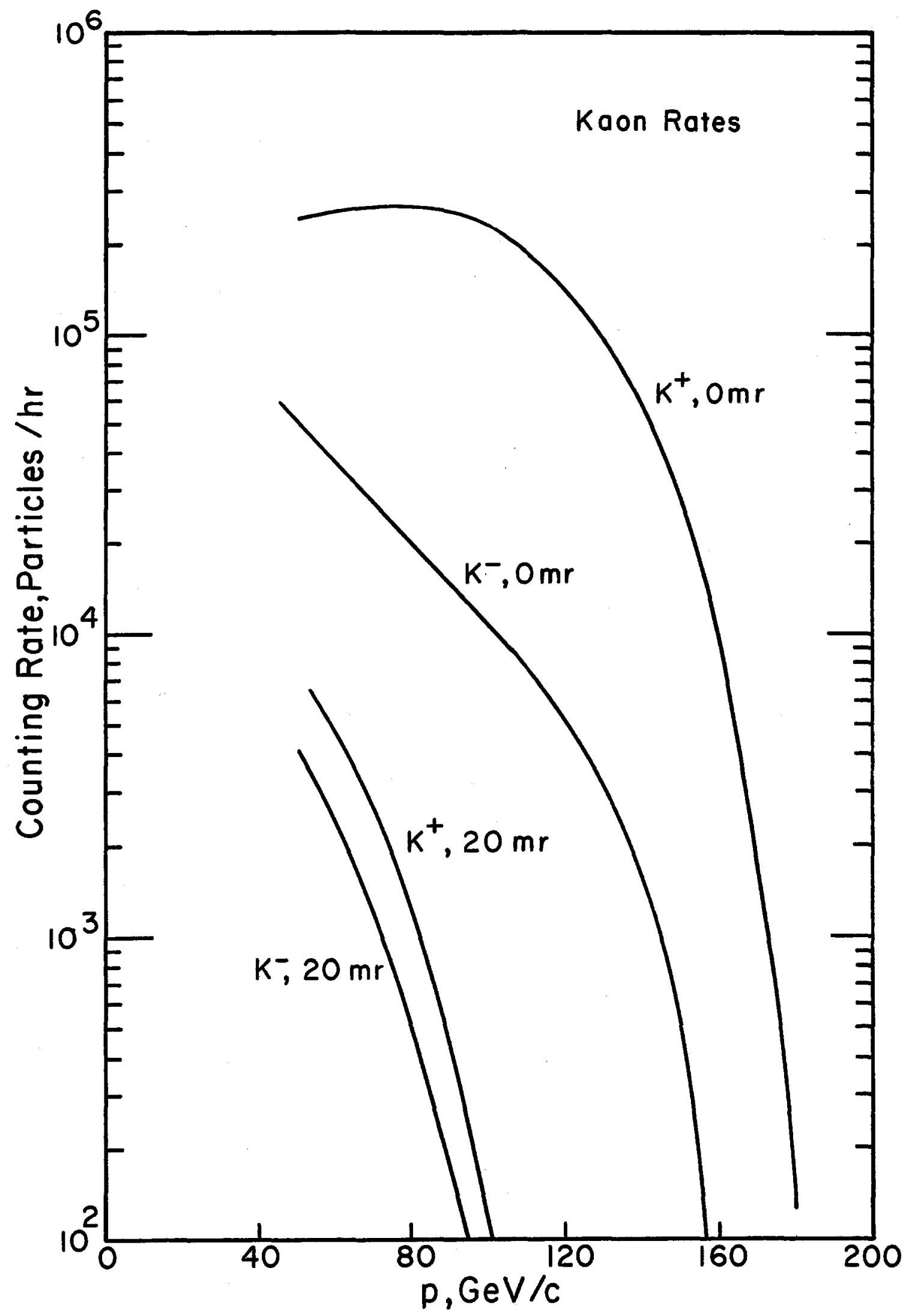

Fig. 4 


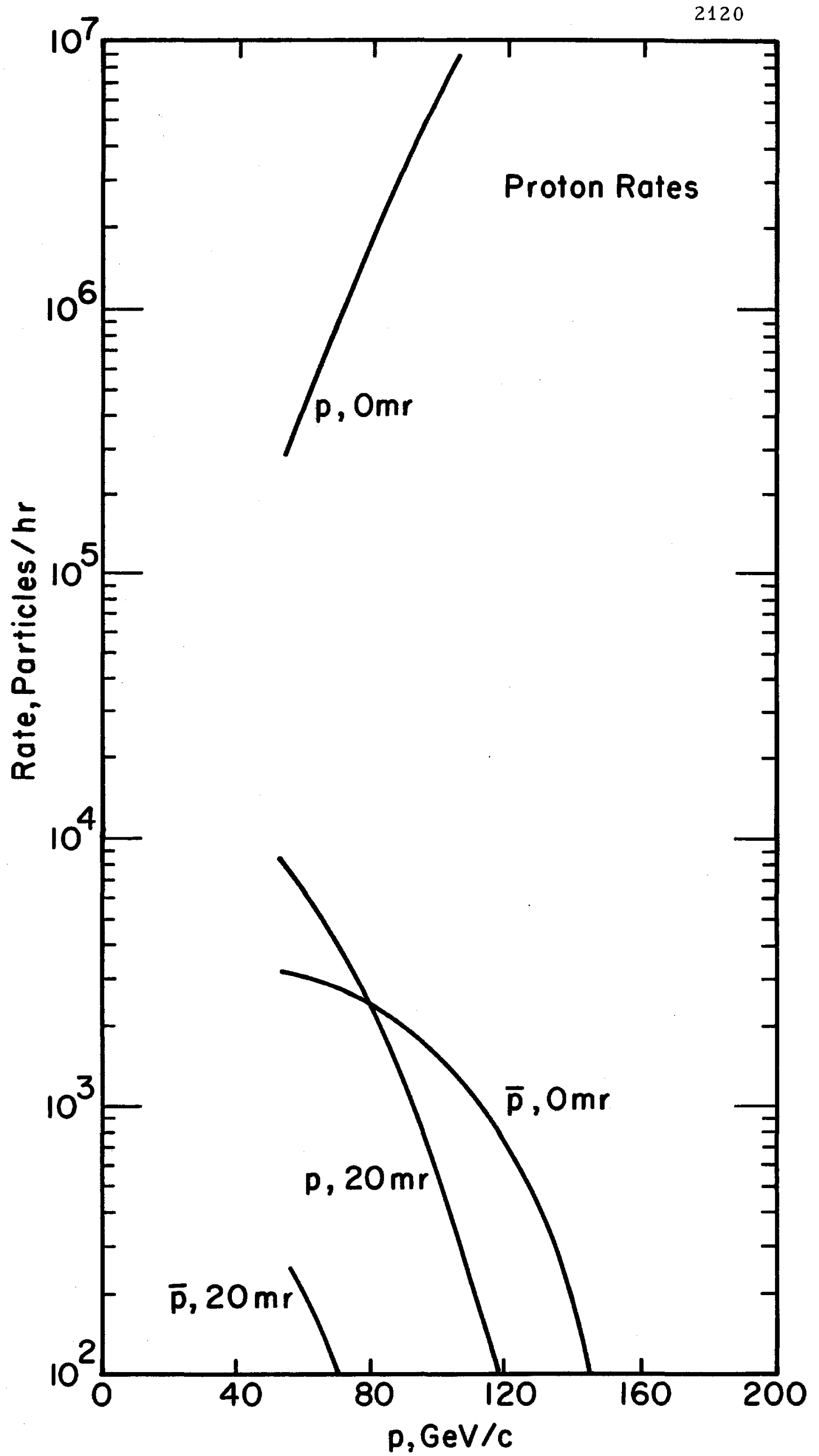

Fio. 5 